\title{
The Reversible Cyclic Universe in the Reversible Multiverse and the Reversible String Theory
}

\author{
Ding-Yu Chung \\ Utica, Michigan, USA \\ Email: dy chung@yahoo.com
}

Received 17 July 2015; accepted 8 August 2015; published 11 August 2015

Copyright (C) 2015 by author and Scientific Research Publishing Inc.

This work is licensed under the Creative Commons Attribution International License (CC BY). http://creativecommons.org/licenses/by/4.0/

(c) (i) Open Access

\begin{abstract}
The paper posits that the multiverse is reversible, so all universes in the multiverse are reversible cyclic universes which have the inexhaustible resources of space-time to expand. The collision of expanding universes is permanently irreversible, forbidden in the reversible multiverse, so every universe is surrounded by the zero-energy interuniversal void as the permanent gap among universes to keep universes apart. A zero-sum energy dual universe of positive energy universe and negative energy universe can be created in the interuniversal void, and the new dual universe is again surrounded by the interuniversal void. This paper also posits the reversible string theory with oscillating space-time dimension number oscillating between 11D (space-time dimension) and 4D without the conventional compactization of string. Dimension number decreases with decreasing speed of light, decreasing vacuum energy, and increasing rest mass. The 4D and the 11D have zero and the highest vacuum energies, respectively. The universes in the reversible multiverse oscillate reversibly between high and low dimension numbers. Under symmetry breaking as in our universe, the positive energy universe as our observed universe absorbed the interuniversal void, while the negative energy universe did not. The interuniversal void has zero vacuum energy, so the absorption of the interuniversal void by the positive energy universe forced the positive energy 10D universe with high vacuum energy to be transformed into the positive energy 4D universe with zero vacuum energy, resulting in the inflation followed by the Big Bang. The negative energy universe undergoes dimension number oscillation between 4D and 10D dimension by dimension. The negative energy $>4 \mathrm{D}$ universe is hidden, and the negative energy $4 \mathrm{D}$ universe appears as dark energy. The calculated percentages of dark energy, dark matter, and baryonic matter and the calculated time for dark energy to start are in good agreements with the observed values.
\end{abstract}

\section{Keywords}

Cyclic Universe, Multiverse, Interuniversal Void, String Theory, Cosmology, Big Bang, Inflation, 


\section{Dark Energy, Dark Matter}

\section{Introduction}

The multiverse has been studied extensively. For example, Brian Greene [1] described the nine types of the multiverse which produce complicated collections of universes. This paper posits a simple version of the multiverse. The posited simple multiverse is the reversible multiverse that excludes any permanently irreversible physical laws and phenomena. In the reversible multiverse, the allowed universes have to be reversible cyclic universes with permanently reversible physical laws and phenomena, resulting in only limited types of allowed universes. Temporary irreversibility is allowed. The paper also posits the reversible physical laws and phenomena are derived from the reversible string theory as described previously [2] [3]. String theory and its extension, membrane theory require the presences of 10 and 11 space-time dimensions, respectively, in contrast to the observed 4D (space-time dimension). In conventional string theory with fixed space-time dimension number, the explanation of the hidden extra space dimensions is the compactization of the extra space dimensions, so space-time appears to be 4D. In the reversible string theory as described previously [2] [3], dimension number oscillates between 11D and 10D and between 10D and 4D reversibly. There is no compactization. The oscillation of space-time dimension number is reversible. Dimension number decreases with decreasing speed of light, decreasing vacuum energy, and increasing rest mass. The 4D particle has the lowest speed of light, zero vacuum energy, and the highest rest mass, while the 11D membrane has the highest speed of light, the highest vacuum energy, and the lowest rest mass. The universes in the multiverse oscillate between high and low dimension numbers.

The reversible cyclic universes have the inexhaustible resources of space-time to expand. In the reversible multiverse, one of the most important exclusions is the exclusion of the collision of expanding universes which cannot be reversed. The exclusion of the collision of expanding universes leads the formation of the "interuniversal voids" that is functioned as the permanently gap among universes to keep universes apart. To prevent the collision, the interuniversal void detaches the incoming mass-energy in the interuniversal void to keep expanding universes apart without collision. When our observed universe absorbed the interuniversal void, the absorbed interuniversal void with the property of the detachment of mass-energy was transformed into the reverse Higgs field (the massless particle generating field) that detached adjacent mass-energy in the universe, resulting in the conversion of rest mass (massive particles) into kinetic energy (massless particles) starting the Big Bang. The absorption of the interuniversal void with zero vacuum energy also started the inflation by converting the 10D universe with high vacuum energy universe into the 4D universe with zero vacuum energy universe. The inflation followed by the Big Bang occurred in our observed universe.

A zero-sum energy dual universe of positive energy universe and negative energy universe can be created in the interuniversal void, and the new dual universe is again surrounded by the interuniversal void. Under symmetry, the new positive and negative energy universes undergo mutual annihilation to reverse to the interuniversal void immediately. Under symmetry breaking as in our universe, the new positive energy universe absorbs the interuniversal void, while the new negative energy does not. With the absorption of the interuniversal void, the positive energy universe as our observed universe had the inflation followed by the Big Bang, while the negative energy universe undergoes dimension number oscillation between 4D and 10D dimension by dimension. The negative energy $>4 \mathrm{D}$ universe is hidden, and the negative energy 4D universe appears as dark energy. As described in this paper, the cyclic universe provides the explanations for the origins of the four force fields (the strong force, the gravity, the electromagnetic force, and the weak force) and dark energy. The calculated percentages of dark energy, dark matter, and baryonic matter and the calculated time for dark energy to start are in good agreements with the observed values.

Section 2 explains the reversible multiverse. Section 3 describes the reversible string theory based on spacetime number oscillation. The reversible cyclic universe derived from the reversible multiverse and the reversible string theory based on oscillating space-time number is explained in Section 4. Section 5 explains the digital space structure derived from the reversible cyclic universe. Section 6 explains the timeline of the physical laws in terms of the force fields, kinetic energy, and quantum fluctuation.

\section{The Reversible Multiverse}

The paper posits that the multiverse is reversible, excluding any permanently irreversible phenomenon. The col- 
lision of expanding universes is permanently irreversible due to the impossibility to reverse the collision of expanding universes, so the collision of expanding universes is excluded. To prevent the collision of expanding universes, every universe is surrounded by the interuniversal void that is functioned as the permanent gap among universes. The interuniversal void has zero energy, zero space-time, and zero vacuum energy, while universe has non-zero energy, the inexhaustible resources of space-time to expand, and zero or/and non-zero vacuum energy. To maintain the permanent gap among universes, the interuniversal void detaches the incoming mass-energy in the interuniversal void, so mass-energy stays permanently within universes only. Surrounded by the interuniversal void as the permanent gap among universes, an expanding universe with the inexhaustible resource of space-time expands without colliding with other universes. The detachment of the incoming mass-energy in the interuniversal void prevents the collision of expanding universes. As a result, the properties of the interuniversal void include the detachment of mass-energy in addition to zero energy, zero space-time, and zero vacuum energy. On the other hand, the properties of universe include the attachment of mass-energy in addition to non-zero energy, the inexhaustible resources of space-time to expand, and zero or/and non-zero vacuum energy. The detachment and the attachment of mass-energy are equivalent to the repulsion and the attraction of mass-energy, respectively. In other words, the interuniversal void repulses (detaches) mass-energy, while universe attracts (attaches) mass-energy.

A zero-sum energy dual universe of positive energy universe and negative energy universe can be created in the zero-energy interuniversal void, and the new dual universe is again surrounded by the interuniversal void to avoid the collision of universes. A dual universe can be created in the interuniversal void, and equally, the "intrauniversal void" can also be created in a universe by the absorption of the interuniversal void. The intrauniversal void can be reversed to the interuniversal void by the desorption of the intrauniversal void. Under symmetry, the new positive energy universe and the new negative energy universe undergo mutual annihilation to reverse to the interuniversal void immediately. Under symmetry breaking with the asymmetrical absorption of the interuniversal void, our dual universe has lasted a long time without reversing immediately to the interuniversal void as described later. The precise definition of the reversibility in the reversible multiverse is the reversibility between universes and the interuniversal void. All universes are created in the interuniversal void, and all universes have to be able to reverse back to the interuniversal void. The parameters involved in the formation of the same type of universes can be different. Reversible symmetry breaking and temporary irreversibility are allowed.

\section{The Reversible String Theory}

The reversible string theory is based on the space-time dimension number oscillation. In the space-time dimension number oscillation, dimension number oscillates between 11D and 10D and between 10D and 4D for the objects, including $11 \mathrm{D}$ membrane $\left(3_{11}\right)$, 10D string $\left(2_{10}\right)$, particle $\left(1_{4}\right.$ to 10$)$, and empty object $\left(0_{4 \text { to } 11}\right)$. Different universes and different stages of a universe can have different expressions of the object structure. Dimension number varies with varying speed of light, vacuum energy, and rest mass. Varying speed of light has been proposed to explain the horizon problem of cosmology [4]. J. D. Barrow [5] proposes that the time dependent speed of light varies as some power of the expansion scale factor $a$ in such way that

$$
c(t)=c_{0} a^{n},
$$

where $c_{0}>0$ and $n$ are constants. The increase of speed of light is continuous.

In this paper, varying dimension number (VDN) relates to quantized varying speed of light (QVSL), where the speed of light is invariant in a constant space-time dimension number, and the speed of light varies with varying space-time dimension number from 4 to 11.

$$
c_{\mathrm{D}}=c / \alpha^{\mathrm{D}-4},
$$

where $c$ is the observed speed of light in the $4 \mathrm{D}$ space-time, $c_{\mathrm{D}}$ is the quantized varying speed of light in space-time dimension number, $\mathrm{D}$, from 4 to 11 , and $\alpha$ is the fine structure constant for electromagnetism. In string theory, the extension of string requires $11 \mathrm{D}$, while the observed space-time dimension is $4 \mathrm{D}$, so $\mathrm{D}$ is from 4 to 11 . Each dimensional space-time has a specific speed of light. (Since from the beginning of our observable universe, the space-time dimension has always been four, there is no observable varying speed of light in our observable universe.) The speed of light increases with the increasing space-time dimension number D. 
In special relativity, $E=M_{0} c^{2}$ modified by Equation (2) is expressed as

$$
\begin{aligned}
E & =M_{0} \cdot\left(c^{2} / \alpha^{2(\mathrm{D}-4)}\right) \\
& =\left(M_{0} / \alpha^{2(\mathrm{~d}-4)}\right) \cdot c^{2} .
\end{aligned}
$$

Equation (3a) means that a particle in the D dimensional space-time can have the superluminal speed $c / \alpha^{\mathrm{D}-4}$, which is higher than the observed speed of light $c$, and has the rest mass $M_{0}$. Equation (3b) means that the same particle in the 4D space-time with the observed speed of light acquires $M_{0} / \alpha^{2(\mathrm{~d}-4)}$ as the rest mass, where $\mathrm{d}=\mathrm{D}$. D from 4 to 11 in Equation (3a) is the space-time dimension number defining the varying speed of light. In Equation (3b), d from 4 to 11 is "mass dimension number" defining varying mass. For example, for D = 11, Equation (3a) shows a superluminal particle in eleven-dimensional space-time, while Equation (3b) shows that the speed of light of the same particle is the observed speed of light with the 4D space-time, and the mass dimension is eleven. In other words, 11D space-time can transform into 4D space-time with 11d mass dimension. 11D4d in Equation (3a) becomes 4D11d in Equation (3b) through QVSL. QVSL in terms of varying space-time dimension number, D, brings about varying mass in terms of varying mass dimension number, d. Mass dimension is also proposed in the Wesson's Space-Time-Matter (STM) theory as the matter dimension to account for the extra space dimensions [6].

The QVSL transformation transforms both space-time dimension number and mass dimension number. In the QVSL transformation, the decrease in the speed of light leads to the decrease in space-time dimension number and the increase of mass in terms of increasing mass dimension number from 4 to 11 ,

$$
\begin{aligned}
& c_{\mathrm{D}}=c_{\mathrm{D}-n} / \alpha^{2 n}, \\
& M_{0, \mathrm{D}, \mathrm{d}}=M_{0, \mathrm{D}-n, \mathrm{~d}+n} \alpha^{2 n}, \\
& \mathrm{D}, \mathrm{d} \stackrel{\mathrm{QVSL}}{\longrightarrow}(\mathrm{D} \mp n),(\mathrm{d} \pm n)
\end{aligned}
$$

where $\mathrm{D}$ is the space-time dimension number from 4 to 11 and $\mathrm{d}$ is the mass dimension number from 4 to 11 . For example, in the QVSL transformation, a particle with 11D4d is transformed to a particle with 4D11d. In terms of rest mass, 11D space-time has 4d with the lowest rest mass, and 4D space-time has 11d with the highest rest mass.

Rest mass decreases with increasing space-time dimension number. The decrease in rest mass means the increase in vacuum energy $\left(E_{\text {vacuum }}=E-M_{0, \mathrm{D}} c^{2}\right)$, so vacuum energy increases with increasing space-time dimension number. The vacuum energy of 4D particle is zero, while $11 \mathrm{D}$ membrane has the Planck vacuum energy.

Since the speed of light for $>4 \mathrm{D}$ particle is greater than the speed of light for 4D particle, the observation of $>4 \mathrm{D}$ superluminal particles by 4D particles violates casualty. Thus, $>4 \mathrm{D}$ particles are hidden particles with respect to $4 \mathrm{D}$ particles. Particles with different space-time dimensions are transparent and oblivious to one another, and separate from one another if possible.

\section{The Reversible Cyclic Universe}

The proposed the cyclic universe starts in the zero-energy interuniversal void, which produces the dual universe of the positive energy $11 \mathrm{D}$ membrane universe and the negative energy $11 \mathrm{D}$ membrane universe denoted as $3_{11} 3_{-11}$, as proposed by Mongan [7]. The only force among the membranes is the pre-strong force, $\mathrm{s}$, as the predecessor of the strong force. It is from the quantized vibration of the membranes to generate the reversible process of the absorption-emission of the particles among the membranes. The pre-strong force mediates the reversible absorption-emission. The pre-strong force is the same for all membranes, so it is not defined by positive or negative sign.

In certain regions of the $11 \mathrm{D}$ membrane universe, the local expansion takes place by the transformation from 11D-membrane into 10D-string. The expansion is the result of the vacuum energy difference between 11D membrane and $10 \mathrm{D}$ string. With the emergence of empty object $\left(0_{11}\right), 11 \mathrm{D}$ membrane transforms into $10 \mathrm{D}$ string with pregravity.

$$
3_{11} s+0_{11} \leftrightarrow 2_{10} s 1_{10}=2_{10} s g^{+}
$$

where $3_{11}$ is the $11 \mathrm{D} 4 \mathrm{~d}$ membrane, $\mathrm{s}$ is the pre-strong force, $0_{11}$ is the $11 \mathrm{D}$ empty object, $2_{10}$ is $10 \mathrm{D} 4 \mathrm{~d}$ string, $1_{10}$ is g, pregravity. Empty object $0_{11}$ corresponds to the anti-De Sitter bulk space in the Randall-Sundrum model [8] 
for gravity. In the other words, pregravity is in the bulk space as the pregravity bulk space. The $\mathrm{g}$ is in the bulk space, which is the warping space (transverse radial space) around $2_{10}$. As in the AdS/CFT duality [9] for gravity, the pre-strong force has 10D dimension, one dimension lower than the 11D membrane, and is the conformal force defined on the conformal boundary of the bulk space.

In the negative universe through symmetry, antistrings form 10D antibranes with anti-pregravity as $2_{-10} g^{-}$, where $g^{-}$is anti-pregravity.

$$
3_{-11} s+0_{-11} \leftrightarrow 2_{-10} s 1_{-10}=2_{-10} s g^{-}
$$

The dual universe of the positive energy 10D string universe with $n$ units of $\left(2_{10}\right)_{n}$ and the negative energy 10D string universe with $\mathrm{n}$ units of $\left(2_{-10}\right)_{n}$ is as follows.

$$
\left(\left(s 2_{10}\right) g^{+}\right)_{n}\left(g^{-}\left(s 2_{-10}\right)\right)_{n}
$$

There are four equal regions: the positive energy $10 \mathrm{D}$ string universe, the pregravity bulk space, the antipregravity bulk space, and the negative energy 10D antistring universe. Such dual universe separated by bulk space appears in the ekpyrotic universe model [10].

Under symmetry, the 10D positive energy string universe and the negative energy 10D string universe coalesce to undergo annihilation and to return to the interuniversal void. Under symmetry breaking as in the case of our universe, the positive energy 10D string universe absorbs the interuniversal void, while the negative energy 10D string universe does not absorb the interuniversal void. The interuniversal void has zero vacuum energy. The absorption of the interuniversal void by the positive energy 10D string universe forces the positive energy 10D universe with high vacuum energy to be transformed into the universe with zero vacuum energy that is the vacuum energy of the $4 \mathrm{D}$ universe. As a result, the positive energy 10D string universe is forced to transform into the positive energy $4 \mathrm{D}$ universe by the absorption of the interuniversal void. However, the transformation is not immediate, because the strings have to be $10 \mathrm{D}$, and it cannot be transformed into $4 \mathrm{D}$, therefore, strings have to be transformed into particles that allow the change of its dimension number freely to accommodate the transformation from the $10 \mathrm{D}$ universe to the $4 \mathrm{D}$ universe driven by the absorption of the interuniversal void. The transformation of strings into particles comes from the emergence of positive pre-charge and negative precharge that allows the mutual annihilation of positively pre-charged 10D strings and negatively pre-charged 10D antistrings in the 10D string universes to produce positively pre-charged 10D particles and negatively precharged 10D antiparticles in the 10D particle universes. The emergence of positive pre-charge and negative precharge provides the prototype of the electromagnetic force with charge.

The emergence of positive and negative pre-charges provides the new distinction between string and antistring. The mixing of string and antistring becomes the mixing of positively pre-charged string and negatively precharged antistring instead of positive energy string and negative energy antistring. The process is the charge transformation as Figure 1.

During the coalescence, the positive energy string and the negative energy antistring intersect at the middle as Figure 1, where string and antistring join and split as in conventional string theory. The combination of one half of the positive energy and one half of the negative energy antistring form a new string. As a result, there are two new strings with the top half and with the bottom half of Figure 1. The top half is transformed into a new positive energy string, while the bottom half is transformed into a new negative energy string. The way to achieve such transformations is through the emergence of pre-charges in the charge transformation. In the top half of the figure above, a positive charge $\left(e^{+}\right)$emerges in the positive energy string, and a negative charge $\left(e^{-}\right)$emerges in the negative energy antistring to transform the negative energy antistring into the positive energy antistring, resulting in the positive energy antistring with negative charge. The new string from the top half includes the positive energy positively pre-charged string and positive energy negatively pre-charged antistring.

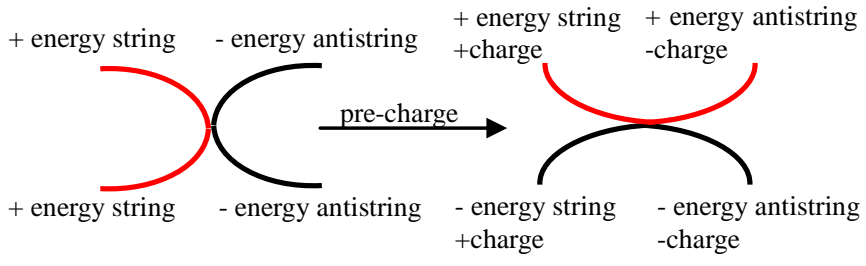

Figure 1. The charge transformation. 
Simultaneously, in the bottom half of the figure above, a negative charge emerges in the negative energy antistring, and a positive charge emerges in the positive energy string to transform the positive energy string into the negative energy string, resulting in the negative energy string with positive charge. The new string from the bottom half includes the negative energy negatively pre-charged antistring and the negative energy positively pre-charged string. In the charge transformation, all transformations and the additions of charges are symmetrical between the two new positive energy and negative energy strings.

The string-antistring annihilation results in the replacement of the 10D string-antistring, $\left(2_{10} 2_{-10}\right)$ by the 10D particle-antiparticle $\left(1_{10} 1_{-10}\right)$. The 10D particles-antiparticles have no fixed space-time dimension number. After the mixing, the dual 10D particle-antiparticle universe separated by pregravity and anti-pregravity appears as below.

$$
\left(\left(s 1_{10} e^{+} e^{-} 1_{-10} s\right) g^{+}\right)_{n}\left(g^{-}\left(s 1_{10} e^{+} e^{-} 1_{-10} s\right)\right)_{n},
$$

where $s$ and $e$ are the pre-strong force and the pre-charged force in the flat space, $g$ is pregravity in the bulk space, and $1_{10} 1_{-10}$ is the particle-antiparticle.

The dual 10D particle universe consists of two parallel particle-antiparticle universes with opposite energies and the bulk space separating the two universes. There are four equal regions: the positive energy particle-antiparticle universe, the pregravity bulk space, the negative energy particle-antiparticle universe, and the anti-pregravity bulk space. The positive energy 10D4d particle universe with high vacuum energy is transformed into the 4D10d universe with zero vacuum energy at once, resulting in the inflation. Without the absorption of the interuniversal void, the negative energy universe starts the gradual expansion from 10D to 4D dimension by dimension, and undergoes the stepwise dimension number oscillation.

At zero vacuum energy, the absorbed interuniversal void is transformed into "the 4D intrauniversal void" with zero vacuum energy and with space-time inside of the universe. Derived from the interuniversal void, the intrauniversal void has the same property of the detachment of mass-energy as the interuniversal void described in Section 2. Inside of the universe, the intrauniversal void with the property of the detachment of mass-energy detaches adjacent mass-energy in the universe, resulting in the conversion of rest mass (massive particles) into kinetic energy (massless particles), so the intrauniversal void is the reverse Higgs field generating massless particles starting the first step of the Big Bang in the universe with the inexhaustible resource of space-time to expand. The Higgs field inside of the universe is desorbed to become the external Higgs field outside of the universe. In terms of the Higgs mechanism, the reverse Higgs field derived from the interuniversal void desorbs the longitudinal component (the Higgs field) inside of the universe to become the external Higgs field outside of the universe, and generated massless particles. The equation for the first step of the Big Bang is as Equation (9)

$$
\text { interuniversal void + Higgs field } \rightarrow \text { reverse Higgs field + external Higgs field }
$$

The diagram for the first step of the Big Bang is Figure 2.

The second step of the Big Bang is the exchange process between the partial absorption of the Higgs bosons (the mediating particles) from the external Higgs field and the partial desorption of the reverse Higgs field inside to become the interuniversal void outside of the universe. Under symmetry breaking, the second step is the partial reversal of the first step. In terms of the Higgs mechanism, the absorbed Higgs bosons provide the longitudinal components for massless particles to form massive particles, and desorbed the reverse Higgs field inside to become the interuniversal void outside to complete the exchange process. Photon without absorbing the Higgs boson remains massless in the partial conversion, and the unabsorbed Higgs boson becomes the observed Higgs boson [11]. The equation for the second step of the Big Bang is as Equation (10).

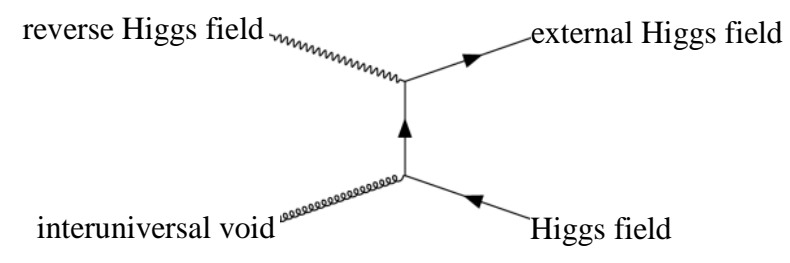

Figure 2. The first step in the Big Bang. 
reverse Higgs field + Higgs boson from the external Higgs field

$\stackrel{\text { partial }}{\longrightarrow}$ Higgs field + interuniversal void

The diagram for the second step of the Big Bang is as in Figure 3.

In summary, the two-step process during the Big Bang included (1) the conversion of rest mass (massive particles) into kinetic energy (massless particles) by the creation of the reverse Higgs field derived from the absorption of the interuniversal void outside of the universe and (2) the partial conversion of massless particles into partial massive particles by the partial absorption of the Higgs bosons from the external Higgs field outside of the universe.

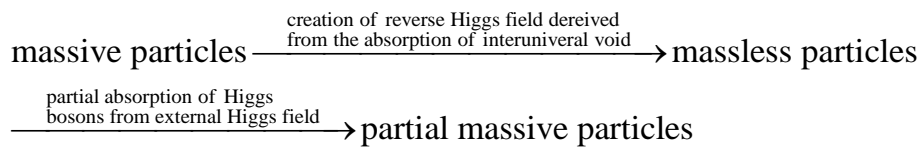

The asymmetrical dual universe consists of the positive-energy 4D universe as the light universe with kinetic energy as light and the negative-energy variable D universe as the dark universe without kinetic energy as light. The asymmetrical dual universe is manifested as the asymmetry in the asymmetrical weak interaction in our observable universe as follows.

Light Universe and bulk space

$$
\left(\left(s 1_{4} e^{+} w^{+} e^{-} w^{-} 1_{-4} s\right) g^{+}\right)_{n}
$$

Dark Universe and bulk space

$$
\left(g^{-}\left(s 1_{4 \text { to } 10} e^{+} w^{+} e^{-} w^{-} 1_{-4 \text { to }-10} s\right)\right)_{n}
$$

where $s, g, e$, and $w$ are the strong force, gravity, electromagnetism, and weak interaction, respectively for the observable universe, and where $1_{4} 1_{-4}$ and $1_{4 \text { to } 10} 1_{-4 \text { to }-10}$ are $4 \mathrm{D}$ particle-antiparticle for the light universe and variable $\mathrm{D}$ particle-antiparticle for the dark universe, respectively.

The dark universe involves the stepwise two-step transformation: the QVSL transformation and the varying supersymmetry transformation from 10D4d to 4D4d. (The particles in the 10D dual particle universe are 10D4d.) The QVSL transformation involves the transformation of space-time dimension, D. The varying supersymmetry transformation involves the transformation of the mass dimension number, d, as follows.

stepwise two-step varying transformation

$$
\begin{aligned}
& \text { (1) } \mathrm{D}, \mathrm{d} \stackrel{\text { Qvarying }}{\stackrel{\mathrm{QVSL}}{\longrightarrow}}(\mathrm{D} \mp 1),(\mathrm{d} \pm 1) \\
& \text { (2) D,d } \stackrel{\text { supersymmetry }}{\longrightarrow} \mathrm{D},(\mathrm{d} \pm 1)
\end{aligned}
$$

The repetitive stepwise two-step transformation from 10D4d to 4D10d as follows.

$$
\begin{aligned}
& \text { 10D4d } \rightarrow \text { 9D5d } \rightarrow \text { 9D4d } \rightarrow \text { 8D5d } \rightarrow \cdots \rightarrow \text { 4D5d } \rightarrow \text { 4D4d } \\
& \mapsto \text { hidden dark } \quad \text { universe } \leftarrow \mapsto \text { dark energy } \leftarrow
\end{aligned}
$$

In this two-step transformation, the transformation from 10D4d to 9D5d involves the QVSL transformation as in Equation (4c).

The dark universe consists of two periods: the hidden dark universe and the dark energy universe. The hidden dark universe composes of the $>4 \mathrm{D}$ particles. As mentioned before, particles with different space-time dimensions are transparent and oblivious to one another, and separate from one another if possible. Thus, $>4 \mathrm{D}$ particles are hidden and separated particles with respect to $4 \mathrm{D}$ particles in the light universe (our observable universe). The hidden dark universe with $\mathrm{D}>4$ and the observable universe with $\mathrm{D}=4$ are the "parallel universes".

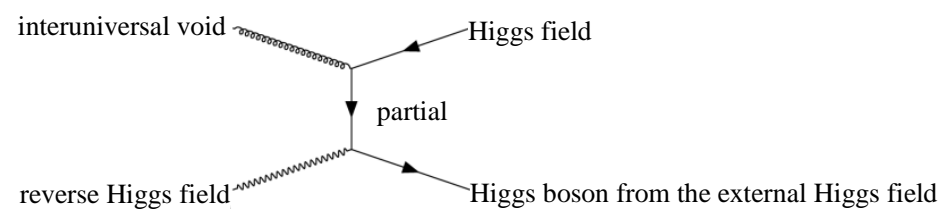

Figure 3. The second step in the Big Bang. 
The 4D particles transformed from hidden $>4 \mathrm{D}$ particles in the dark universe are observable dark energy for the light universe, resulting in the accelerated expanding universe. Since the dark universe without kinetic energy, the presence of dark energy is not different from the presence of the cosmological constant. In terms of quintessence, such dark energy can be considered the tracking quintessence [12] from the dark universe with the space-time dimension number as the tracker. Observable dark energy emerged about 5 billion years ago (more precisely $4.71 \pm$ 0.98 billion years ago at $z=0.46 \pm 0.13$ [13]).

The four regions in the light-dark dual universe include the light universe, the gravity bulk space, the antigravity bulk space, and the dark universe. Through the symmetry among the space regions, all regions expand synchronically and equally. (The symmetry is necessary for the ultimate reversibility in the reversible multiverse.) The light universe is the only region with the four-dimensional space-time and with kinetic energy from the beginning, and all other three regions have variable dimensional space-time without kinetic energy. The light universe occupies $25 \%$ of the total universe, while the other regions occupy $75 \%$ of the total universe, so the maximum dark energy from the dark universe, the gravity bulk space, and the antigravity bulk space is $75 \%$. The present observable universe about reaches the maximum (75\%) at the observed $72.8 \%$ dark energy [14]. According to the calculation from the previous paper [3], dark energy started in 4.28 billion years ago in agreement with the observed $4.71 \pm 0.98$ billion years ago.

After the maximally connected universe, $4 \mathrm{D}$ dark energy transforms back to $>4 \mathrm{D}$ particles that are not observable. The removal of dark energy in the observable universe results in the stop of accelerated expansion and the start of contraction of the observable universe. The end of dark energy starts another "parallel universe period”. Both hidden universe and observable universe contract synchronically and equally. Eventually, the Big Crush-deflation occurs in the light universe to reverse to the positive energy 10D particle universe. Meanwhile, the dark universe is transformed into the negative energy 10D particle universe. The dual universe can undergo another cycle of the light-dark dual universe. On the other hand, both universes can undergo the reverse charge transformation to become the 10D dual string universe, which in turn can return to the 11D dual membrane universe that in turn can return to the interuniversal void as in Figure 4.

\section{The Digital Space Structure}

The multiverse can be considered as the digital multiverse consisting of +1 for positive energy universe, -1 for negative energy universe, and 0 for the interuniversal void. The sum in the digital multiverse is 0 , so the multiverse is the reversible digital zero-sum multiverse. During the Big Bang, the combination of the positive energy universe as 1 and the interuniversal void as 0 in the digital multiverse led to the digital space structure consisting of attachment space (the Higgs field) as 1 and detachment space (the reverse Higgs field) as 0 in the positive energy universe as in Equation (15).

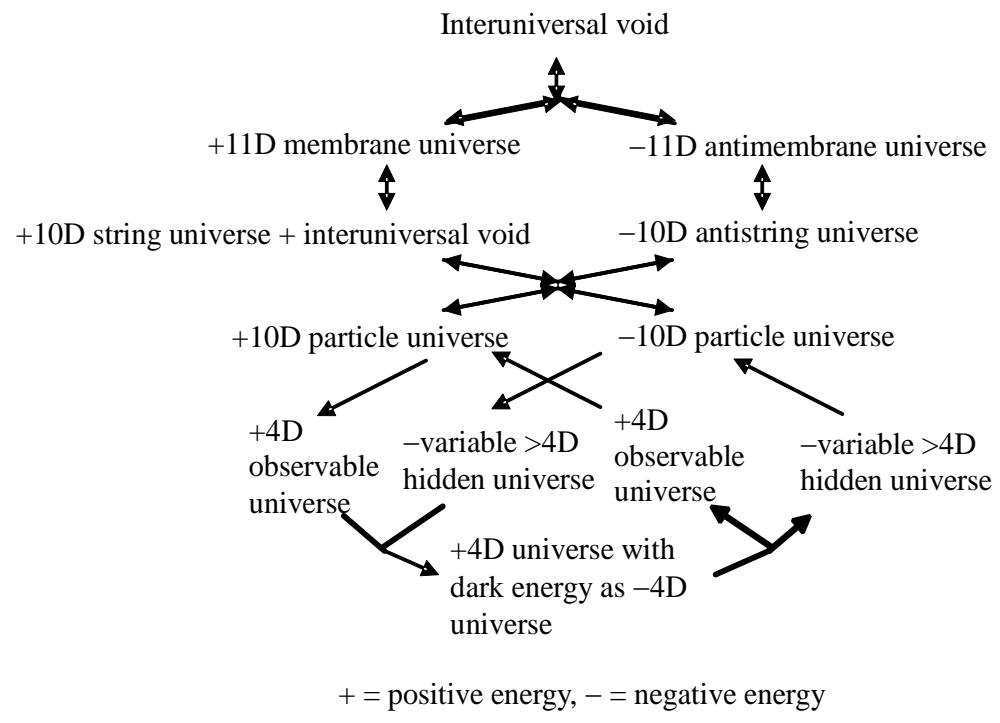

Figure 4. The reversible cyclic universe. 
positive energy universe(1)+ interuniversal void(0)

$\rightarrow$ attachment space as Higgs field(1) + detachment space as reverse Higgs field $(0)$

The digital space structure is described in the previous paper [15] [16]. Derived from the space of the universe before the Big Bang, attachment space is the Higgs field, and attaches to mass-energy continuously and reversibly. Derived from the interuniversal void outside of the universe, detachment space is the reverse Higgs field, and detaches adjacent mass-energy continuously. Attachment space relates to massive particles, rest mass and reversible movement, while detachment space relates to massless particles and irreversible kinetic energy adjacent to detachment space continuously.

The combination of $n$ units of attachment space as 1 and $n$ units of detachment space as 0 brings about three different space structures: binary partition space, miscible space, or binary lattice space as below.

$$
(1)_{n} \quad+\quad(0)_{n} \stackrel{\text { combination }}{\longrightarrow}(1)_{n}(0)_{n}, \quad(1+0)_{n}, \quad \text { or } \quad(10)_{n}
$$

attachment space detachment space binary partition space, miscible space, binary lattice space

Binary partition space, $(1)_{n}(0)_{n}$, consists of two separated continuous phases of multiple quantized units of attachment space and detachment space. In miscible space, $(1+0)_{n}$, attachment space is miscible to detachment space, and there is no separation of attachment space and detachment space. Binary lattice space, $\left(\begin{array}{ll}1 & 0\end{array}\right)_{n}$, consists of repetitive units of alternative attachment space and detachment space.

Binary partition space is the space for wavefunction in quantum mechanics. In wavefunction,

$$
|\Psi\rangle=\sum_{i=1}^{n} c_{i}\left|\phi_{i}\right\rangle
$$

Each basis element, $\left|\phi_{i}\right\rangle$, has both attachment space and detachment space as binary partition space. Neither attachment space nor detachment space is zero in binary partition space for a basic element. The measurement in the uncertainty principle in quantum mechanics is essentially the measurement of attachment space size and momentum from the detachment space in binary partition space: large momentum from detachment space has small non-zero attachment space size, while large attachment space size has low non-zero momentum from detachment space. In binary partition space, an entity is both in constant motion as wave for detachment space and in stationary state as a particle for attachment space, resulting in the wave-particle duality.

In binary partition space, for every detachment space, there is its corresponding adjacent attachment space. Thus, no part of the mass-energy can be irreversibly separated from binary partition space, and no part of a different mass-energy can be incorporated in binary partition space. Binary partition space represents coherence as wavefunction. Binary partition space is for coherent system. Any destruction of the coherence by the addition of a different mass-energy to the mass-energy causes the collapse of binary partition space into miscible space. The collapse is a phase transition from binary partition space to miscible space.

$$
\begin{aligned}
& (0)_{n}(1)_{n} \stackrel{\text { collapse }}{\longrightarrow}(0+1)_{n} \\
& \text { binary partition space miscible space }
\end{aligned}
$$

The information in miscible space is contributed by the miscible combination of both attachment space and detachment space, so information can no longer be non-localized. Any value in miscible space is definite. All observations in terms of measurements bring about the collapse of wavefunction, resulting in miscible space that leads to eigenvalue as definite quantized value. Such collapse corresponds to the appearance of eigenvalue, $E$, by a measurement operator, $H$, on a wavefunction, $\Psi$.

$$
H \Psi=E \Psi
$$

In miscible space, attachment space is miscible to detachment space, and there is no separation of attachment space and detachment space. In miscible space, attachment space contributes zero speed, while detachment space contributes the speed of light. For a moving massive particle consisting of a rest massive part and a massless part, the massive part with rest mass, $m_{0}$, is in attachment space, and the massless part with kinetic energy, $K$, is adjacent to detachment space. The combination of the massive part in attachment space and massless part in detachment leads to the propagation speed in between zero and the speed of light. To maintain the speed of light constant for a moving particle, the time $(t)$ in moving particle has to be dilated, and the length $(L)$ has to be contracted relative to the rest 
frame.

$$
\begin{aligned}
& t=t_{0} / \sqrt{1-v^{2} / c^{2}}=t_{0} \gamma, \\
& L=L_{0} / \gamma, \\
& E=K+m_{0} c^{2}=\gamma m_{0} c^{2}
\end{aligned}
$$

where $\gamma=1 /\left(1-v^{2} / c^{2}\right)^{1 / 2}$ is the Lorentz factor for time dilation, and length contraction, $E$ is the total energy, and $K$ is the kinetic energy.

Bounias and Krasnoholovets [17] propose that the reduction of dimension can be done by slicing dimension, such as slicing 3 space dimension object (block) into infinite units of 2 space dimension objects (sheets). As described in Section 4 [2] [3], the positive energy 10D4d particle universe as our observed universe with high vacuum energy was transformed into the 4D10d universe with zero vacuum energy at once, resulting in the inflation. During the Big Bang following the inflation, the 10d (mass dimension) particle in attachment space denoted as 1 was sliced by detachment space denoted as 0 . For example, the slicing of $10 \mathrm{~d}$ particle into $4 \mathrm{~d}$ particle is as follows.

$$
1_{10} \stackrel{\text { slicing }}{\longrightarrow} 1_{4} \quad \sum_{\mathrm{d}=5}^{10}\left(0_{4} 1_{4}\right)_{n, \mathrm{~d}}
$$

10d particle 4d core particle binary lattice space

where $1_{10}$ is $10 \mathrm{~d}$ particle, $1_{4}$ is $4 \mathrm{~d}$ particle, $\mathrm{d}$ is the mass dimension number of the dimension to be sliced, $\mathrm{n}$ as the number of slices for each dimension, and $\left(0_{4} 1_{4}\right)_{n}$ is binary lattice space with repetitive units of alternative $4 \mathrm{~d}$ attachment space and $4 \mathrm{~d}$ detachment space. For $4 \mathrm{~d}$ particle starting from $10 \mathrm{~d}$ particle, the mass dimension number of the dimension to be sliced is from $\mathrm{d}=5$ to $\mathrm{d}=10$. Each mass dimension is sliced into infinite quantized units $(n=\infty)$ of binary lattice space, $\left(0_{4} 1_{4}\right)_{\infty}$. For $4 d$ particle, the $4 d$ core particle is surrounded by 6 types (from $d=5$ to $d=10$ ) of infinite quantized units of binary lattice space. Such infinite quantized units of binary lattice space represent the infinite units $(n=\infty)$ of separate virtual orbitals in a gauge force field, while the dimension to be sliced is "dimensional orbital" (DO), representing a type of gauge force field. The mass-energy in each dimensional orbital increases with the number of dimension number, and the lowest dimension orbital with $d=5$ has the lowest mass-energy [11] [18].

10d particle was sliced into six different particles: 9d, 8d, 7d, 6d, 5d, and 4d equally by mass. Baryonic matter is $4 \mathrm{~d}$, while dark matter consists of the other five types of particles (9d, 8d, 7d, 6d, and 5d). The mass ratio of dark matter to baryonic matter is 5 to 1 . At $72.8 \%$ dark energy, the calculated values for baryonic matter and dark matter (with the $1: 5$ ratio) are $4.53 \%(=(100-72.8) / 6)$ and $22.7 \%(=4.53 \times 5)$, respectively, in excellent agreement with observed $4.56 \%$ and $22.7 \%$, respectively [14]. The dimensional orbitals of baryonic matter provide the base for the periodic table of elementary particles to calculate accurately the masses of all elementary particles, including quarks, leptons, gauge bosons, the Higgs boson, and the knees-ankles-toe in cosmic rays [11] [18] [19].

The lowest dimensional orbital is for electromagnetism. Baryonic matter with maximum number of gauge force fields (dimensional orbitals) is the only one with the lowest dimensional orbital for electromagnetism. With higher dimensional orbitals, dark matter does not have this lowest dimensional orbital [15]. Without electromagnetism, dark matter cannot emit light, and is incompatible to baryonic matter with electromagnetism, like the incompatibility between oil and water. Derived from the incompatibility between dark matter and baryonic matter, the modified interfacial gravity (MIG) between homogeneous baryonic matter region and homogeneous dark matter region to separate baryonic matter region and dark matter region explains galaxy evolution and unifies the CDM (Cold Dark Matter) model, MOG (Modified Gravity), and MOND (Modified Newtonian Dynamics) [20] [21].

The space structure based on the combination of binary partition space and binary lattice space explains superconductivity [22] and superstar without singularity to replace black hole with singularity [16] [23]. Singularity is permanently irreversible by losing information permanently, forbidden in the reversible multiverse.

\section{The Timeline of the Physical Laws}

Conventionally, the physical laws in terms of the force fields, kinetic energy, and quantum fluctuation have always existed. In the cyclic universe proposed in this paper, the physical laws appear and disappear in different stages of the universe. The physical laws in different stages of the universe are linked, so a physical law in one 
stage provides the prototype of a physical law in the subsequent stage. In the cyclic universe as shown in Figure 4 , the force field in each of the four different universes, the positive energy 11D membrane universe, 10D string universe, 10D particle universe, and 4D particle universe, provided the prototype of each of the four different observed force fields, the strong force, gravity, the electromagnetic force, and the weak force, respectively, in the observed universe.

The force field among the membranes in the 11D membrane universes provided the prototype of the strong force without charge in our observed universe. The observed strong force was created during the Big Bang as a force field in binary lattice space around particles. The gravity in the bulk space of the 10D string universes provided the prototype of observed gravity as warping space in the observed universe. The cyclic universe consists of equal four regions: the positive energy universe (the observed universe), the gravity bulk space, the antigravity bulk space, and the negative energy universe. The observed gravity as warping space around particles was created during the Big Bang. Gravity in the bulk space is observed only as a part of dark energy, when the bulk space reaches $4 \mathrm{D}$.

The absorption of the interuniversal void by the positive energy10D string universe forced the 10D universes to be transformed from the 10D universes into the 4D universes, so strings had to be transformed into particles that allowed the change of its dimension number freely to accommodate the transformation from the 10D universe to the 4D universe driven by the absorption of the interuniversal void. The transformation of strings into particles came from the emergence of positive charge and negative charge that allowed the mutual annihilation of positively charged 10D strings and negatively charged 10D antistrings in the 10D string universes to produce positively charged 10D particles and negatively charged 10D antiparticles in the 10D particle universes. The force field involved in positive charge and negative charge provided the prototype of the observed electromagnetic force with charge. The observed electromagnetic force in binary lattice space around particles was created during the Big Bang. The force field to represent the asymmetry between the positive energy $4 \mathrm{D}$ particle universe and the negative energy variable $\mathrm{D}$ particle universe provided the prototype of the observed asymmetrical weak force in the observed universe. The observed weak force in binary lattice space around particles was created during the Big Bang. As the result of the causality relationship between the 10D particle universe and the $4 \mathrm{D}$ particle universe, the electromagnetic force and the weak force were unified symmetrically as the electroweak force during the Big Bang. The symmetry breaking in the electroweak force occurred during the second step in the Big Bang.

Irreversible kinetic energy and quantum fluctuation were created during the Big Bang. There were no irreversible kinetic energy and quantum fluctuation before the Big Bang. There will be no irreversible kinetic energy and quantum fluctuation after the Big Crush. Before the Big Bang and after the Big Crush, all parts of the cyclic universe are completely reversible and homogeneous because of the complete reversibility in the reversible multiverse. After the Big Bang and before the Big Crush, the observed universe allows temporary irreversibility from irreversible kinetic energy and temporary inhomogeneity from quantum fluctuation.

\section{Summary}

The paper posits that the multiverse is reversible, so all universes in the multiverse are reversible cyclic universes which have the inexhaustible resources of space-time to expand. The collision of expanding universes is permanently irreversible, forbidden in the reversible multiverse, so every universe is surrounded by the zero-energy interuniversal void as the permanent gap among universes to keep universes apart. A zero-sum energy dual universe of positive energy universe and negative energy universe can be created in the interuniversal void, and the new dual universe is again surrounded by the interuniversal void. This paper also posits the reversible string theory with oscillating space-time dimension number oscillating between 11D (space-time dimension) and 4D without the conventional compactization of string. Dimension number decreases with decreasing speed of light, decreasing vacuum energy, and increasing rest mass. The 4D and the 11D have zero and the highest vacuum energies, respectively. The universes in the reversible multiverse oscillate reversibly between high and low dimension numbers. Under symmetry breaking as in our universe, the positive energy universe as our observed universe absorbed the interuniversal void, while the negative energy universe did not. The interuniversal void has zero vacuum energy, so the absorption of the interuniversal void by the positive energy universe forced the positive energy 10D universe with high vacuum energy to be transformed into the positive energy 4D universe with zero vacuum energy, resulting in the inflation followed by the Big Bang. The negative energy un- 
iverse undergoes dimension number oscillation between 4D and 10D dimension by dimension. The negative energy $>4 \mathrm{D}$ universe is hidden, and the negative energy $4 \mathrm{D}$ universe appears as dark energy. The calculated percentages of dark energy, dark matter, and baryonic matter and the calculated time for dark energy to start are in good agreements with the observed values.

\section{References}

[1] Greene, B. (2011) The Hidden Reality: Parallel Universes and the Deep Laws of the Cosmos. Vintage, New York.

[2] Chung, D. and Krasnoholovets, V. (2013) Journal of Modern Physics, 4-7A, 77-84.

[3] Chung, D. (2014) Journal of Modern Physics, 5, 464-472. http://dx.doi.org/10.4236/jmp.2014.56056

[4] Albrecht, A. and Magueijo, J. (1999) Physics Review, D59, Article ID: 043516. http://dx.doi.org/10.1103/PhysRevD.59.043516

[5] Barrow, J.D. (2003) Physics Letter, B564, 1-7. http://dx.doi.org/10.1016/S0370-2693(03)00573-2

[6] Wesson, P.S. (1999) Space-Time-Matter: Modern Kaluza -Klein Theory. World Scientific Publishing Company, Singapore.

[7] Mongan, T.R. (2005) General Relativity and Gravitation, 37, 967-970. http://dx.doi.org/10.1007/s10714-005-0079-y

[8] Randall, L. and Sundrum, R. (1999) Physical Review Letters, 83, 4690-4693. http://dx.doi.org/10.1103/PhysRevLett.83.4690

[9] Maldacena, J. (1998) Advance Theoretic Mathematics Physics, 2, 231-252.

[10] Steinhardt, P.J. and Turok, N. (2002) Physical Review, D65, Article ID: 126003. http://dx.doi.org/10.1103/PhysRevD.65.126003

[11] Chung, D.Y. and Hefferlinm, R. (2013) Journal of Modern Physics, 4-4A, 21-26.

[12] Padmanabhan, T. (2003) Physical Report, 380, 235-320. http://dx.doi.org/10.1016/S0370-1573(03)00120-0

[13] Riess, A.G., Strolger, L.G., Tonry, J., Casertano, S., Ferguson, H.C., Mobasher, B., et al. (2004) The Astrophysical Journal, 607, 665-687. http://dx.doi.org/10.1086/383612

[14] Jarosik, N., Bennett, C.L., Dunkley, J., Gold, B., Greason, M.R., Halpern, M., et al. (2011) The Astrophysical Journal Supplement Series, 192, 14. http://dx.doi.org/10.1088/0067-0049/192/2/14

[15] Chung, D.Y. and Krasnoholovets, V. (2013) Journal of Modern Physics, 4-4A, 27-31.

[16] Chung, D.Y. (2014) Global Journal of Science Frontier Research A, 14-3, 1-8.

[17] Bounias, M. and Krasnoholovets, V. (2003) The International Journal of Systems and Cybernetics, 32, 1005-1020.

[18] Chung, D.Y. (2014) Journal of Modern Physics, 5, 1234-1243. http://dx.doi.org/10.4236/jmp.2014.514123

[19] Chung, D.Y. (2014) Journal of Modern Physics, 5, 1467-1472. http://dx.doi.org/10.4236/jmp.2014.515148

[20] Chung, D.Y. (2014) International Journal of Astronomy and Astrophysics, 4, 374-383. http://dx.doi.org/10.4236/ijaa.2014.42032

[21] Chung, D.Y. (2015) Global Journal of Science Frontier Research A, 15-3, 119-125.

[22] Chung, D.Y. (2015) Journal of Modern Physics, 6, 26-36. http://dx.doi.org/10.4236/jmp.2015.61005

[23] Chung, D.Y. and Krasnoholovets, V. (2013) Journal of Modern Physics, 4-7A, 1-6. 